\section{AB0739 MULTISYSTEM INFLAMMATORY SYNDROME ASSOCIATED WITH SARS-COV-2 INFECTION AND E.COLI SEPSIS: THE POTENTIAL ROLE OF PROCALCITONINE AS A RAPID DIAGNOSTIC BIOMARKER TO DISTINGUISH TWO DIFFERENT PHASES OF SYSTEMIC INFLAMMATORY RESPONSE SYNDROME}

M. Gilio ${ }^{1}$, S. B. Morella ${ }^{1}$, F. Picaro ${ }^{1}$, C. Acierno ${ }^{1}$, D. Palazzo ${ }^{1}$, A. Erezanu ${ }^{1}$ M. Frontuto ${ }^{1}$, G. Mastroberti ${ }^{1}$, G. De Stefano ${ }^{1} .{ }^{1}$ San Carlo Hospital, Infectious Diseases, Potenza, Italy

Background: Severe acute respiratory syndrome coronavirus 2 (SARS-CoV-2) infection is typically very mild and often asymptomatic in children. A complication is the rare multisystem inflammatory syndrome in children (MIS-C) associated with COVID-19, presenting 4-6 weeks after infection as high fever, organ dysfunction, and strongly elevated markers of inflammation. The pathogenesis is unclear but has overlapping features with Kawasaki disease suggestive of vasculitis and a likely autoimmune etiology.

Objectives: We report a case of multisystem inflammatory syndrome in children (MIS-C) in patient with SARS-CoV-2 infection and Enteropathogenic Escherichia coli (EPEC) sepsis due to acute enteritis, observed at end of December 2020 to a tertiary-care center (San Carlo Hospital), in Basilicata region (Italy).

Methods: This healthy 12-year- old male patient was tested positive for severe acute respiratory syndrome coronavirus 2 (SARS-CoV-2) infection. Clinical presentations was characterized by fever, abdominal pain, gastrointestinal complaints and evanescent rash. Laboratory values were remarkable for high levels of procalcitonin, C-reactive protein (CRP), D-dimers, B-type natriuretic peptide (BNP), and troponin. He also had low albumin levels. Autoantibodies tests were negative. Chest tomography showed ground-glass opacities in less than $25 \%$ of the lungs, small bilateral pleural effusion and increased cardiac area; abdominal tomography showed enlargement of the lymphnodes and ascites. Evaluation for other infectious etiologies showed molecular test positivity on fecal samples for EPEC E. coli. He received broad spectrum intravenous antibiotics (macrolids and quinolones and then carbapenems). On the seventh day the enteritis resolved and procalcitonin normalized, however he continued to have lymphopenia, thrombocytopenia, hypoalbuminemia, elevated levels of CRP, D-dimers, ferritin, troponin, and increased BNP. On the ninth day he was feverish again and developed severe cardiac and respiratory failure requiring advanced respiratory support and admission to the intensive care unit. He received IVIG (intravenous immunoglobulin at $2 \mathrm{~g} / \mathrm{Kg}$, glucocorticoids (Methylprednisolone $1 \mathrm{mg} / \mathrm{kg}$ ) and enoxaparin.

Results: The patient was discharged asymptomatic at home after 28 days of hospital stay.

Conclusion: We observed multisystem inflammatory syndrome in children (MISC) in a previously healthy patient with SARS-CoV-2 infection and E.coli sepsis, who became critically ill with multisystem involvement. In this case viral and bacterial infections could be considered as a double hit for the etiopathogenesis of MIS-C. The trend of procalcitonin was better than C-reactive protein for differentiating bacterial from non-bacterial phase of systemic inflammatory response syndrome (SIRS) in this critically ill child. Although the accuracy of both tests is moderate. Diagnostic accuracy could be enhanced by combining these tests with bedside clinical judgment.

REFERENCES:

[1] Consiglio CR, Cotugno N, Sardh et al. The Immunology of Multisystem Inflammatory Syndrome in Children with COVID-19. Cell. 2020 Nov 12;183(4):968-981.e7. doi: 10.1016/j.cell.2020.09.016. Epub 2020 Sep 6. PMID: $32966765 ;$ PMCID: PMC7474869.

[2] Nakra NA, Blumberg DA, Herrera-Guerra A, Lakshminrusimha S. Multi-System Inflammatory Syndrome in Children (MIS-C) Following SARS-CoV-2 Infection: Review of Clinical Presentation, Hypothetical Pathogenesis, and Proposed Management. Children (Basel). 2020 Jul 1;7(7):69. doi: 10.3390/ children7070069. PMID: 32630212; PMCID: PMC7401880.

[3] Simon L, Saint-Louis P, Amre DK, Lacroix J, Gauvin F. Procalcitonin and C-reactive protein as markers of bacterial infection in critically ill children at onset of systemic inflammatory response syndrome. Pediatr Crit Care Med. 2008 Jul;9(4):407-13. PMID: 18496408

Disclosure of Interests: None declared

DOI: 10.1136/annrheumdis-2021-eular.3300

\section{$\mathrm{AB} 0740$ \\ IMPACT OF DIAGNOSIS DELAY ON DISEASE PARAMETERS DURING JUVENILE IDIOPATHIC ARTHRITIS}

H. Bettaieb ${ }^{1}$, H. Ferjani ${ }^{1}$, K. Maatallah ${ }^{1}$, D. Kaffel ${ }^{1}$, W. Hamdi ${ }^{1} .{ }^{1}$ Mohamed Kassab Institute of Orthopedics, Rheumatology, Tunis, Tunisia
Background: Juvenile idiopathic arthritis (JIA) is the most common type of arthritis in childhood (1). Prompt diagnosis is mandatory to avoid joint destruction and growth abnormalities. However, it's often misdiagnosed by pediatricians and general practitioners leading to longer diagnosis delay (2).

Objectives: The aim of this study was to evaluate the lag time between JIA symptoms onset and diagnosis and its impact on disease activity and bone loss. Methods: A retrospective monocentric study was carried out on JIA patients (ILAR criteria). Diagnosis delay was collected from the patient's medical files. Disease activity at JIA diagnosis was evaluated by JADAS10 (Juvenile Arthritis Disease Activity Score) in poly and oligoarticular subtypes and by BASDAI (Bath Ankylosing Spondylitis Disease Activity Index) in arthritis related enthesitis form. The data were analyzed using the SPSS statistical package. A $p$ value $<0.05$ was considered significant.

Results: We enrolled $48 \mathrm{JIA}$ (31 male and 17 female) with a mean age at disease onset of $11.2 \pm 3.8$ years. The median disease duration was 84 months [2-408]. The median JIA diagnosis delay was 8 months [1-108]. The JIA subgroups were in decreasing order of frequency: Enthesitis-related Arthritis $(n=32)$, Polyarticular RF- $(n=4)$, Polyarticular RF+ $(n=2)$, Oligoarticular $(n=6)$, Systemic $(n=2)$, Psoriatic Arthritis $(n=1)$ and Undifferentiated $(n=1)$.

At diagnosis, median ESR and CRP were $44 \mathrm{~mm} / \mathrm{hour}$ [2-100] and $24 \mathrm{mg} / \mathrm{l}[2-86]$ respectively. Median JADAS10 score was 4 [0-21]. Median BASDAI score was $6.2[2-9.4]$.

At follow-up, five patients (10.4\%) had atlantoaxial subluxation and 17 had coxitis $(43.8 \%)$.

At bone densitometry, $45 \%$ of patients had osteroposis and $27.5 \%$ had osteopenia.

An agreement was assessed between a long diagnosis delay and the following parameters: male gender $(p=0.04)$ and osteoporosis $(p=0.018)$. A Significant positive correlation was found between delay in JIA diagnosis and BASDAI score $(p=0.047, r=0.63)$. No association was found between $\mathrm{JIA}$ diagnosis delay and JADAS score $(p=0.56)$. Neither ESR $(p=0.19)$ nor CRP $(p=0.42)$ was associated with JIA diagnosis delay.

Finally, no link was observed with the occurrence of hip arthritis $(p=0.281)$ or atlantoaxial subluxation $(p=0.137)$.

Conclusion: In this study, delay in diagnosis was associated with higher disease activity scores and bone loss. Our results suggest that early identification and treatment of JIA leads to improved outcomes as well as bone mass.

\section{REFERENCES:}

[1] Petty R.E., Southwood T.R., Manners P. International League of Associations for Rheumatology classification of juvenile idiopathic arthritis: second revision, Edmonton, 2001. J Rheumatol. 2004;31(2):390.

[2] Foster HE, Scott C, Tiderius CJ,et al. Improving musculoskeletal health for children and young people - A 'call to action' Best Pract Res Clin Rheumatol. 2020 Oct;34(5):101566.

Disclosure of Interests: None declared

DOI: 10.1136/annrheumdis-2021-eular.3472

\section{AB0741 PREVALENCE AND ASSOCIATED FACTORS OF ATLANTO-AXIAL INSTABILITY IN PATIENTS WITH JUVENILE IDIOPATHIC ARTHRITIS}

H. Bettaieb ${ }^{1}$, H. Ferjani ${ }^{1}$, K. Maatallah ${ }^{1}$, H. Boussaa ${ }^{1}$, D. Kaffel ${ }^{1}$, W. Hamdi ${ }^{1}$.

'Mohamed Kassab Institute of Orthopedics, Rheumatology, Tunis, Tunisia

Background: Atlanto-axial instability (AAI) is a serious complication during Juvenile Idiopathic Arthritis (JIA). It can lead to severe neurological morbidity or mortality if left untreated (1).

Objectives: To determine the prevalence of AAI in patients with JIA and to identify factors associated with an increased risk of its occurrence.

Methods: A retrospective monocentric study was carried out on JIA patients (ILAR criteria). Data, including age at disease onset, JIA type, disease activity at AAI diagnosis and treatment were collected. Disease activity at JIA diagnosis was evaluated by JADAS10 (Juvenile Arthritis Disease Activity Score) in poly and oligoarticular subtypes and by BASDAI (Bath Ankylosing Spondylitis Disease Activity Index) in arthritis related enthesitis form. Standard radiographs of the cervical spine were analyzed. The data were analyzed using the SPSS statistical package. A p value $<0.05$ was considered significant.

Results: We enrolled $48 \mathrm{JIA}$ ( 31 male and 17 female) with a mean age at disease onset of $11.2 \pm 3.8$ years. The median disease duration was 84 months [2-408]. The median JIA diagnosis delay was 8 months [1-108]. The JIA subgroups were in decreasing order of frequency: Enthesitis-related Arthritis $(n=32)$, Polyarticular RF- $(n=4)$, Polyarticular RF+ $(n=2)$, Oligoarticular $(n=6)$, Systemic $(n=2)$, Psoriatic Arthritis $(n=1)$ and Undifferentiated $(n=1)$. 